\title{
Efectos pleiotrópicos de las estatinas
}

\author{
Dr. Benjamín Stockins \\ Facultad de Medicina Universidad de La Frontera \\ Recibido el 12 de julio de 2009. Aceptado el 20 de julio 2009
}

Rev Chil Cardiol 2009; 28: 198-201

El artículo de Alcaíno y colaboradores ${ }^{1}$ coloca nuevamente en el tapete el tema de los efectos hipolipemiantes y pleiotrópicos de las estatinas. La aparición de estos fármacos, obtenidas a partir de hongos Aspergillus, significó una revolución en el terreno del tratamiento hipolipemiante y de ese modo en la prevención primaria y secundaria de la enfermedad aterosclerótica. La evidencia de la correlación entre niveles de colesterol y enfermedad coronaria en poblaciones aborígenes y occidentales, así como en primates y mamíferos superiores, es abrumadora, no cabiendo duda de su importancia como factor de riesgo cardiovascular 2.

Las estatinas han mostrado una potentísima capacidad de reducir los niveles de colesterol y también el de reducir eventos en forma linear a la reducción de dichos niveles.

Sin embargo, y sin desconocer la acción hipolipemiante de estos fármacos, las estatinas han agregado un nuevo concepto, el cual no ha estado exento de polémicas. Ello consistió en incorporar a estos medicamentos una acepción venida desde la genética, el pleiotropismo o expresión fenotípica múltiple. En relación con las estatinas, este efecto fue definido como "la capacidad de actuar sobre otros procesos relacionados con la ateroescelerosis, mas allá de los lípidos y lipoproteínas" 3 .

Lo anterior ha sido consecuencia de la aparición progresiva de hechos, en un comienzo sugerentes y luego de evidencias, de efectos que van más allá de la reducción del colesterol, ya sea por la temporalidad de los eventos, como por una menor correlación con la reducción del colesterol. Uno de los primeros indicios se dio en la acción de estos fármacos en el Síndrome Coronario Agudo, en él que la reducción de los efectos alcanzó una precocidad no explicable por la acción del colesterol en la reducción de la placa ateroesclerótica. Los estudios MIRACL 4 y PROVE IT5, realizados en pacientes con Síndromes Coronarios Agudos, mostraron que los eventos cardiovasculares presentaban una disminución significativa tras escasas semanas de tratamiento, a lo que se asoció una disminución de parámetros de inflamación, como la Proteína C Reactiva Hipersensible.

Un análisis posterior del estudio WOSCOPS 6 también demostró una disminución significativa de eventos con la terapia con pravastatina al ajustar para los niveles lipídicos encontrados.

Otras observaciones que apoyan un beneficio adicional son la disociación entre angiografía y efectos clínicos (poco cambio en arterias), el beneficio clínico mayor a la modificación del colesterol, la posible mejoría de otras patologías con estatinas (osteoporosis, cáncer) y la reducción de episodios de accidente vascular cerebral isquémico (patología poco relacionada con los niveles de colesterol) ${ }^{7}$.

Tras ello se han planteado numerosas acciones benéficas de estos fármacos como son 8,9 :

- La estabilización de la placa de ateroma, al reducir el nivel de métalo proteinazas en ella. Disminución en el contenido de lípidos y macrófagos y aumentar el grosor de la cápsula.

- Modulación de la función endotelial, por un incremento en la producción de Oxido Nítrico y una

Correspondencia: Dr. Benjamín Stockins

Facultad de Medicina Universidad de La Frontera

Temuco - Teléfono: (45) 245753

Correo Electrónico: stockins@ufro.cl 
reducción de la síntesis y acción de la endotelina y angiotensina II.

- Efectos antinflamatorios, al disminuir marcadores de inflamación como PCR, Interleukina 6 y, necrosis tumoral alfa. Con ello reducen la migración y proliferación de los monolitos.

- Efectos antioxidantes: Los radicales libres aumentan la oxidación del Colesterol LDL y aumentan la aterogénesis. También favorecen la migración de monocitos a macrófagos y son responsables de la ruptura de células espumosas. Las estatinas reducen la expresión de radicales libres y preservan la capacidad antioxidante.

- Efectos antitrombóticos: Las estatinas reducen la agregación plaquetaria, la concentración de fibrinógeno y la viscosidad sanguínea.

- Efectos sobre la angiogénesis, al estimular la expresión del factor de movilización de células madre del endotelio desde la médula ósea.

El mecanismo que explicaría estos efectos "más allá" del colesterol, o efectos pleiotrópicos, estaría relacionado con la inhibición de una de las primeras etapas de la formación del colesterol, el paso de HMG-CoA a ácido mevalónico, así como la inhibición de $\mathrm{RHO}$ y de $\mathrm{RHO}$ quinasa 10 .

La existencia de estos efectos no ha estado exenta de controversias, existiendo autores que estiman que todos ellos son secundarios a su acción hipolipemiante, sobre la base de que siempre se asocian a alguna acción sobre los lípidos y a que dichos beneficios pueden ser atribuidos a la reducción del colesterol LDL.

Hoy en día apoyan esta independencia de acción diversos hechos, como la rapidez de su efecto, el haber sido efectivas en pacientes homocigotos, en los que no cambian los niveles de colesterol y los estudios en modelos animales en los que las estatinas no modifican los niveles de colesterol, pero si aumentan la biodisponibilidad de óxido nítrico, inhiben la hipertrofia ventricular y el tamaño de accidentes vasculares cerebrales.
Como muchos medicamentos de acciones variadas se ha intentado ver sus efectos benéficos en otras patologías relacionadas. Una de ellas fue la estenosis aórtica ${ }^{11}$ cuyos resultados fueros desalentadores y la otra es la insuficiencia cardíaca. Respecto a esta última existen varios estudios cuyos resultados han sido más bien negativos en cuanto a su utilidad en reducir eventos clínicos.

Los estudios en insuficiencia cardíaca partieron de la lógica de que las estatinas serían beneficiosas en esta condición dada su acción a nivel vascular. Ello se vio avalado por estudios pequeños y de otros metodológicamente impugnables. Dos grandes estudios que intentaron responder a esta preguntar corresponden al CORONA 12 y al del grupo GISSI-HF13. CORONA incorporó a 5.011 pacientes con insuficiencia cardíaca en capacidad funcional II a IV, los que fueron randomizados a rosuvastatina o placebo, y seguidos en un promedio de 32.8 meses. Aún cuando existió una reducción significativa del colesterol en el grupo tratado, ello no se tradujo en ningún beneficio en los parámetros definidos por el estudio (muerte de cualquier causa, evento coronario, muerte cardiovascular, infarto del miocardio no fatal o accidente vascular cerebral no fatal). El estudio GISSI-HF fue realizado en 4.574 pacientes con insuficiencia cardíaca, los que fueron randomizados igualmente a rosuvastatina o placebo, y seguidos por un promedio de 3.9 años. Al cabo de dicho período no se encontró una diferencia en los parámetros estudiados (tiempo hasta la muerte o hasta la admisión a un hospital por causa cardiovascular).

El artículo de Alcaíno y colaboradores ${ }^{1}$ da una nueva visión en este aspecto en una serie pequeña, pero muy bien estudiada de pacientes con insuficiencia cardíaca (en su mayor parte de origen no isquémico). En ella se midió la acción de atorvastatina sobre los niveles de la enzima prooxidante xantina-oxidasa unida al endotelio, en pacientes portadores de este síndrome en un período de ocho semanas. Sus resultados muestran una reducción significativa de la enzima en comparación con placebo. Interesantemente, no se apreció una relación significativa entre la fracción de cambio de la enzima y la fracción de cambio del colesterol total, lo que sugiere una acción independiente de estos fármacos sobre la 
enzima, respecto a la reducción del colesterol.

Se trata de un trabajo original cuyas limitaciones están dadas por el número de pacientes y por tratarse de un diseño no ciego. Sin embargo, muestran una línea de investigación que puede dar interesantes frutos a futuro.

Podemos decir que hoy en día hemos evolucionado a un concepto en el cual existe un proceso dinámico en la pared arterial y en el cual el advenimiento de las estatinas ha jugado un rol primordial. Hoy en día nadie duda de sus efectos colaterales sobre la función endotelial, la inflamación y la trombosis.

Existe controversia en relación a efectos pleiotrópicos de otros hipolipemiantes como el ezetimibe, los fibratos y la niacina. El primero ha tenido resultados contradictorios y estudios pequeños no han mostrado una mejoría de la función endotelial al ser comparados con una estatina ${ }^{14}$. Respecto a los otros, sus resultados son de una época en la cual todo estaba centrado alrededor del descenso del colesterol (a plazos más largos), por lo que no existen datos sólidos al respecto 15 .

En resumen, la acción pleiotrópica de las estatinas es un concepto atractivo que permite explicar efectos colaterales a su acción hipolipemiante. Sin embargo es aún imposible separar acciones independientes de estos fármacos de un efecto reducidor de los niveles de colesterol LDL.

Sin embargo ante la ausencia de evidencia definitiva, el mundo se divide entre los "creyentes y no creyentes del pleiotropismo"16. Los primeros estiman que las acciones anteriormente expuestas no son consecuencia directa de la reducción de los niveles de colesterol y que su administración produce una mejoría independiente. Los segundos atribuyen todas sus virtudes a la reducción de los niveles de colesterol.

Sobre dicha base no debemos olvidar que nuestro blanco fundamental continúa siendo la reducción del colesterol LDL.

La verdad última al respecto no está dicha, sin embargo no existen dudas en que las estatinas, los fármacos más prescritos a nivel mundial, juegan y seguirán jugando un rol sustantivo en la reducción de eventos cardiovasculares, tanto precoces como tardíos.

\section{Referencias}

1. ALCAINO H, CASTRO P, VERDEJO H, CHIONG M, GARCIA L, GABRIELLI L, et al. El tratamiento con Atorvastatina reduce la actividad de la xantina-oxidasa unida al endotelio en pacientes con insuficiencia cardíaca crónica: ¿Un posible nuevo efecto pleiotrópico?. Rev Chil Cardiol 2009; 28: 139-149

2. CASTELLI WP. Epidemiology of coronary heart disease. Am J Med 1984; 76: 4-12

3. DAVIDSON M. Clinical significance of statin pleiotropic effects. Circulation 2005; 111: 2280-281

4. SCHWARTZ GG, OLSSON AG, EZEKOWITZ MD GANZ P, OLIVER MF, WATERS D, et al. Effects of atorvastatin on early recurrent ischemic events in acute coronary syndromes: the MIRACL study: a randomized controlled trial. JAMA 2001; 285: 1711-1718

5. RAY KK, CANNON CP, MCCABE CH, CAIRNS R, TONKIN AM, SACKS FM, et al. Early and late benefits of high-dose atorvastatin in patients with acute coronary syndromes: results from the PROVE IT-TIMI 22 trial. J Am Coll Cardiol 2005; 46: 1405-1410

6. West of Scotland Coronary Prevention Group. Influence of pravastatin and plasma lipids on clinical events in the West of Scotland Coronary Prevention Study (WOSCOPS). Circulation 1998;97: 1436-39

7. O'REGAN C, WU P, ARORA P, PERRI D, MILLS EJ. Statin therapy in stroke prevention: a metaanalisis

involving 121.000 patients. Am J Med 2008; 121: 24-33

8. ARTEAGA A, VERDUGO C. Estatinas: Efectos extralipídicos. Lípidos y Ateroesclerosis. Editorial Bywaters, 2005: 83-91.

9. DAVIGNON J. Beneficial cardiovascular pleiotropic effects of statins. Circulation 2004; 109; III-39-III-43

10. MENNICKENT CS, BRAVO DM, CALVO MC, AVELLO LM. Pleiotropic effects of statins. Rev Med Chil 2008; 136: 775-782

11. ROSSEBO AB, PEDERSEN TR, BOMAN K, BRUDI P, CHAMBERS JB, EGSTRUP $\mathrm{K}$, et al. Intensive lipid lowering with simvastatin and ezetimibe in aortic stenosis. N Engl J Med 2008 359: 1343-56

12. KJEKSHUS J, APETREI E, BARRIOS V, BÖHM M, CLELAND JG, CORNEL JH, et al. Rozuvastatin in older 


\section{B. Stockins}

patients with systolic heart failure. N Eng J Med 2007; 357: 2248-61

13. TAVAZZI L, MAGGIONI AP, MARCHIOLI R, BARLERA S, FRANZOSI MG, LATINI R, et al. Effects of rozuvastatin in patients with chronic heart failure (the GISSI-HF trial): a randomized, double blind, placebo-controlled trial. Lancet 2008; 372: 1231-9

14. LANDMESSER U, BAHLMANN F, MUELLER M,
SPIEKERMANN S, KIRCHHOFF N, SCHULZ S, et al. Simvastatin versus Ezetimibe: pleiotropic and lipid-lowering effects on endothelial function in humans. Circulation 2005; 111: 2356-2363

15. SHAW S, FILDES J, YONAN N ET AL. Pleiotropic effects and cholesterol - lowering therapy. Cardiology 2009; 112: 4-12

16. LANSBERG P. Pleiotropic effects of statins and beyond. Cardiology 2009; 112: 1-3 